\title{
On Technical Change in the Elasticities of Resource Inputs
}

J. Growiec and I. Schumacher

Discussion Paper 2006-31

\section{Département des Sciences Économiques de l'Université catholique de Louvain}




\title{
CORE DISCUSSION PAPER 2006/63
}

\section{On Technical Change in the Elasticities of Resource Inputs*}

\author{
Jakub Growiec $^{\dagger} \quad$ Ingmar Schumacher ${ }^{\ddagger}$
}

June 29,2006

*The first author acknowledges financial support from the EDNET Marie Curie fellowship program. The second author acknowledges financial support from the Chair Lhoist Berghmans in Environmental Economics and Management. We are grateful to Thierry Bréchet and Stéphane Lambrecht for their useful comments.

${ }^{\dagger}$ CORE, Université Catholique de Louvain, 34 Voie du Roman Pays, 1348 Louvain-la-Neuve, Belgium; and Warsaw School of Economics, Warsaw, Poland. E-mail address: growiec@core.ucl.ac.be

${ }^{\ddagger}$ Corresponding author. CORE, Université Catholique de Louvain, 34 Voie du Roman Pays, 1348 Louvain-la-Neuve, Belgium. E-mail address: schumacher@core.ucl.ac.be 


\begin{abstract}
This article considers an economy whose production function takes both renewable and non-renewable resources as inputs. We extend the current literature by allowing for exogenous technical change in the elasticity of substitution between these two types of resources. In addition, we study the consequences of biased technical change which alters the resources' relative productivities. We derive long-run asymptotic results, which we use to compare several cases. In the benchmark case of no technical change, our results are close to those obtained by Dasgupta and Heal (1974). In the case of technical change in the elasticity of substitution, we observe that this kind of technical change helps obtain positive long-run production despite the depletion of non-renewable resources. In the biased technical change case, longrun production is only possible either if non-renewable resources are non-essential or if biased technical change is quick enough to compensate for the decreasing flow of non-renewable resources.

We embed our production function in an optimal growth model and study its dynamics. As a steady state (or a balanced growth path) is only attainable as time goes to infinity, we resort to numerical simulations to convey what is happening during the short and medium run. Our results provide new considerations for the debate on natural resources. We suggest that technical change should be directed to the resource which is most important for production.
\end{abstract}

Keywords: Elasticity of substitution, Technical change, Biased technical change, Nonrenewable resources, Renewable resources.

JEL Classification: Q20, Q30, O30. 


\section{Contents}

1 Introduction $\quad 1$

2 Technical change in the CES function $\quad 3$

2.1 CES and changing elasticity of substitution . . . . . . . . . . . . 4

2.2 CES and biased technical change . . . . . . . . . . . . . . 6

2.3 Closing the model . . . . . . . . . . . . . . . . . . . . . 9

3 Asymptotic results $\quad 11$

3.1 Flexibility is the key . . . . . . . . . . . . . . . . 11

3.2 Increasing flexibility saves the day . . . . . . . . . . . . . . . . 12

3.3 Biased technical change . . . . . . . . . . . . . . . . 13

4 The Hotelling model revisited $\quad 15$

4.1 The model . . . . . . . . . . . . . . . . . . . 15

4.2 Comparative statics . . . . . . . . . . . . . . . . . 17

4.3 Simulations and analysis . . . . . . . . . . . . . . . 18

5 Conclusion $\quad 24$

$\begin{array}{ll}\text { A Appendix } & 27\end{array}$

A.1 Normalization of the CES function family . . . . . . . . . . . . . 27

A.2 Asymptotic results for the partial elasticities . . . . . . . . . . . . . . . 29

A.3 Optimization of the Hotelling model . . . . . . . . . . . . . . . . . 29

A.4 Proof that the denominator of $\hat{R}_{N}$ is always positive . . . . . . . . . . . 30

$\begin{array}{ll}\text { References } & 31\end{array}$ 


\section{Introduction}

The sheer size of today's population coupled with its endless desires imposes a never known demand on the production of goods and services. The world's GDP has never been as high as now, and with the advent of China's and India's economic expansions, it seems clear that world production is bound to increase substantially. A noticeable problem is, however, that production depends on various inputs, some of which are known to be limited as well as non-renewable. This has raised concerns by major institutions (notably the RFF, World Bank) for the possibility of continuing production at today's levels to the further future. As pointed out in a special issue of the Review of Economic Studies already back in the 70's, the ability to extend our current opportunities to future generations, when production utilizes limited, non-renewable resources, depends on a variety of factors, of which two have been singled out as the most crucial ones: substitutability and technical change.

Substitutability is vital because, as Dasgupta and Heal (1974) show, non-renewable resources are essential for production if other inputs are poor substitutes for them. For example, insulation can reduce the amount of oil necessary for heating, but cannot be a perfect substitute for oil. Empirical evidence for low substitution possibilities is provided by Cleveland and Ruth (1997). If this is actually the case, this strand of research predicts a bleak future for generations to come.

Technical change, either exogenous as in Solow (1956) or endogenous as in Romer (1990), is the other factor vital for the further understanding of production processes. As an example, one could think of cars which are developed such that they can do the same mileage with less and less petrol. Equipped with these new growth-theoretic tools, resource economists (e.g. Scholz and Ziemes, 1999; Groth, 2004; Bretschger, 2005) return with the hope that, even under initially unfavorable circumstances, production possibilities would not decline due to human ingenuity. These theories teach us that the general requirement for non-declining production is a fast enough technical change (Schou, 2000; Bretschger, 2005). However, it is again Cleveland and Ruth (1997, p. 217) who forcefully argue that "...technology and substitution have not been sufficiently strong to offset the effects of depletion at the macroeconomic scale in some nations."

Our article extends the literature in several ways. Whereas the classic works (Solow, 
1974; Stiglitz, 1974; Dasgupta and Heal, 1974) mainly analyze the relationship between capital and non-renewable resources, we are going to concentrate on the relationship between non-renewable and renewable resources in production. We shall use a production function of the constant elasticity of substitution (CES) type to allow for various degrees of substitutability between the two inputs. This generalization is necessary as we don't have strong empirical evidence of the level of substitutability between non-renewable and renewable inputs. Furthermore, this approach follows recent research by André and Cerdá (2005) as well as Grimaud and Rouge (2005). Our analysis, however, takes a different direction to theirs. We are allowing for technical change in the actual elasticity of substitution. This permits us to gain new insights into short-run and long-run dynamics. To our knowledge, this approach is novel to the literature.

The extension of technical change in the elasticity of substitution does not just come out of nowhere. Demands to analyze the effect of technical change on the elasticity of substitution have been raised time and again during the past 70 years, with stronger and clearer requests. Already Hicks, who invented the concept of the elasticity of substitution in the 30's, points out that the elasticity of substitution might change because "(...) methods of production already known, but which did not pay previously, may come into use." Even more precise, he suggests that substitution "(...) partly takes place by affording a stimulus to the invention of new types." (1932, p.120) De La Grandville then proposes to think about the elasticity of substitution as "a measure of the efficiency of the productive system" (1989, p.479), which is something that is going to become clear during the following sections. Yuhn, who tests de La Grandville's proposition, suggests to view the elasticity of substitution as "a 'menu of choice' available to entrepreneurs." (1991, p. 344) All of these researchers believe the elasticity of substitution to be a decisive component, if not a determinant, of growth. They furthermore suggest that the elasticity is by no means invariable over time. That there might exist technical innovations driving changes in the elasticity of substitution has recently been put forward by Klump and Preissler (2000, p.52), when they suggest that "[a]s far as invention of new methods of production is concerned, the elasticity of substitution as a measure of economic progress can, of course, be related to a society's capability to create and maintain a high rate of innovative activities." Finally, and most precisely, Bretschger (2005, p.150) suggests that "all possibilities of substitution and, specifically, the effects technology exerts on 
promoting substitution have to be studied." Among others, these points quoted here provide a foundation for introducing technical change in the elasticity of substitution.

Moreover, we also introduce biased technical change in the partial elasticities of the renewable and non-renewable resources. This means that both resource inputs are subject to technical change and therefore increase their productivity, but one of the resources, in our case the renewable one, is subject to quicker technical change than the other resource. This allows us to draw conclusions that go beyond those present in the established literature.

Our article is structured as follows. Section 2 introduces the implications of allowing for technical change in the elasticity of substitution and in the distribution parameter of a CES function with non-renewable and renewable resources inputs. We use this function in section 3 in a global production function and derive asymptotic results that allow us to draw long-run conclusions. Section 4 derives optimality conditions for an infinite horizon Ramsey-Hotelling economy which is based on our production function. As a steady state or a balanced growth path is generally not possible, we resort to numerical simulations when explaining the results that we obtain. Section 5 concludes.

\section{Technical change in the CES function}

We shall use the by now common constant elasticity of substitution (CES) production function, as derived in the seminal article by Arrow et al. (1961), and allow for technical change in the elasticity of substitution, or alternatively, in the distribution parameter of the CES function.

Thus, we shall assume the following. The intermediate resource input $R(t)$ is produced with flows of the non-renewable resource $R_{N}(t) \geq 0$, and the renewable resource $R_{R}(t) \geq$ 0 . They are combined according to:

$$
R(t)=\left[\psi(t) R_{N}(t)^{\theta(t)}+(1-\psi(t)) R_{R}(t)^{\theta(t)}\right]^{\frac{1}{\theta(t)}},
$$

where the distribution factor is given by $\psi(t) \in[0,1]$, and the elasticity of substitution, 
$\sigma(t) \in(0,+\infty)$, is related to the elasticity parameter $\theta(t)$ via

$$
\theta(t)=\frac{\sigma(t)-1}{\sigma(t)}
$$

At each instant of time, flows of resources used up in production diminish their stocks. The non-renewable resource stock $0<S_{N}(0)<\infty$ is gradually depleted according to $\dot{S}_{N}(t)=-R(t)<0^{1}$, while the stock of the renewable resource $S_{R}(t)$, evolves according to $\dot{S}_{R}(t)=\varpi\left(S_{R}(t)\right)-R_{R}(t){ }^{2}$

The elasticity of substitution $\sigma(t)$ and the distribution parameter $\psi(t) \in[0,1]$ are allowed to change over time, but not simultaneously. ${ }^{3}$

\subsection{CES and changing elasticity of substitution}

The elasticity of substitution $\sigma(t)$ gives the percentage change in relative quantities of used resources given a one per-cent change in their relative prices ${ }^{4}$. If $\sigma(t)=0$ then the

\footnotetext{
${ }^{1}$ Throughout the article we shall use, for any variable $B(t), \dot{B}(t)$ to denote the time derivative of $B(t)$, $\hat{B}(t)$ to denote its growth rate. For any function $G(B), G^{\prime}(B)$ denotes its first derivative, and $G^{\prime \prime}(B)$ its second derivative with respect to $B$.

${ }^{2}$ We assume that the function $\varpi$ is first increasing, then decreasing, and concave on its whole domain, $\varpi^{\prime \prime}\left(S_{R}(t)\right)<0$. We also assume that once completely exhausted renewable resource does not renew itself any more, $\varpi(0)=0$, and that there exists a unique $\bar{S}_{R}>0$ such that $\varpi\left(\bar{S}_{R}\right)=0$. These assumptions have been borrowed from Smulders (1995), and are consistent with some simple notions from the laws of thermodynamics. They are not necessary for the rest of the article; we quote them here since they may help the reader agree with our subsequent assumptions.

${ }^{3}$ There is an important caveat here. Since we want to obtain meaningful results and yet allow the elasticity of substitution to change over time, we have to normalize the family of CES functions under consideration (de La Grandville, 1989; Klump and Preissler, 2000). To keep things as simple as possible, we note that the Arrow et al. (1961) way of writing a CES function family, with the elasticity parameter being the only parameter allowed to change, implies that this family is normalized at the level $r_{0}=$ $1, n_{0}=1$. We can pick such baseline values without loss of generality, but we are not allowed to manipulate the distribution parameter at the same time. Conversely, when we allow for technical change in the distribution parameter, normalization forces us to assume constancy of the elasticity of substitution. Having this remark in mind, we clearly see that our approach is consistent with normalization, i.e. we do not confuse "intra-" and "inter-family" differences between CES functions (Klump and Preissler, 2000). A more formal and elaborate discussion of this point has been included in Appendix A.1.

${ }^{4}$ This is equivalent to assuming perfect markets, so that price of each input $i$ is always equal to the marginal product of input $i$.
} 
function is Leontief so the inputs are perfect complements, for $\sigma(t)=1$ we obtain the standard Cobb-Douglas form, and if $\sigma(t)=\infty$ then the function is linear so the inputs are perfect substitutes. The elasticity of substitution therefore gives information on the ease with which one can move along a given isoquant and in that way can either be understood as a measure of flexibility, efficiency (de La Grandville, 1989), or 'menu of choice' (Yuhn, 1991). We shall view the elasticity of substitution as a measure of technical efficiency. ${ }^{5}$ As can be seen in Figure 1, the higher the elasticity of substitution, the larger the amount

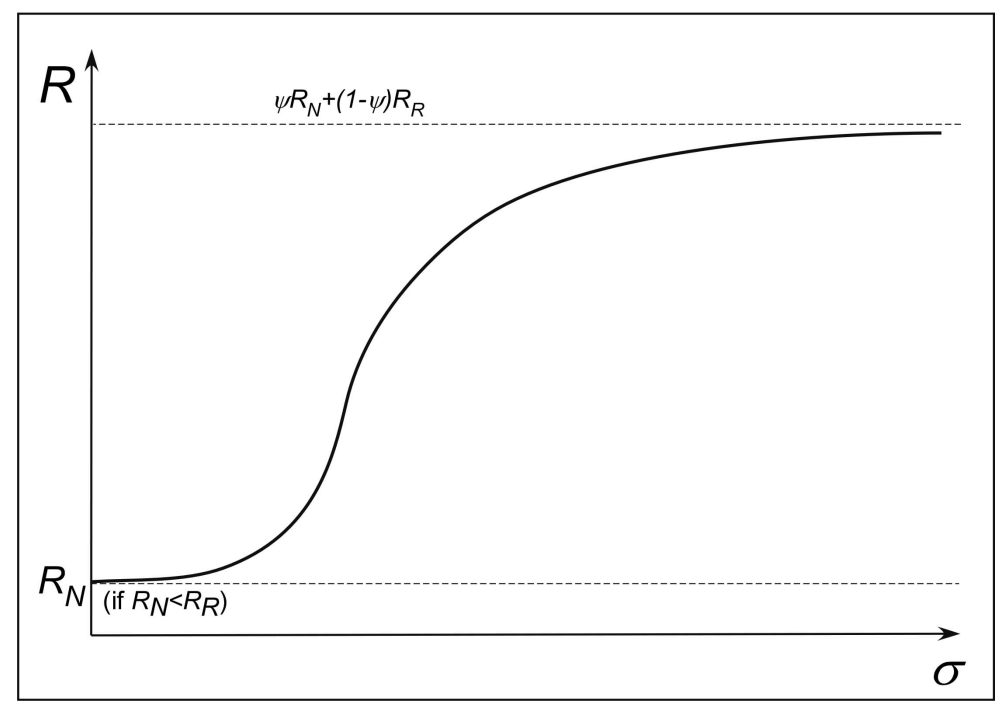

Figure 1: $R$ as a function of $\sigma$

of output $R(t)$ for any given inputs $R_{N}(t)$ and $R_{R}(t)$. The intermediate resource input production is a monotonic function of the elasticity of substitution, $\frac{\partial R(t)}{\partial \sigma(t)}>0$, and follows a convex-concave shape.

The elasticity pulls double-duty here. On the one hand it is a measure of efficiency, and on the other it determines the essentiality of the inputs. If $\sigma(t) \leq 1$, then $R(t) \rightarrow 0$ if any of the two inputs goes to zero. On the contrary, if $\sigma(t)>1$, neither of the inputs is

\footnotetext{
${ }^{5}$ For example, if one wants to produce shoes, then one needs leather and rubber. Imagine that one has a cow, which can provide leather for many shoes, and enough rubber for just one sole. Then, to produce this one shoe, one has to kill the cow. Clearly, this is not very efficient. If one could however produce the shoes with more flexibility, one could substitute the leather for the scarce rubber and thus make many shoes and not waste the rest of the leather.
} 
essential any longer.

As suggested in the Introduction, technical change can be thought of as improving upon the efficiency of the input usage. We shall assume that this is actually the case and study exogenous technical change in the elasticity of substitution, given as follows:

$$
\sigma(t)=\sigma(0) e^{s t}, \quad \sigma(0)>0,
$$

such that the growth rate of $\sigma(t)$ is $s \geq 0$. Thus, whatever the initial relationship between our inputs, they will eventually become perfect substitutes. This assumption does not seem to be far fetched. For example, the recently invented biodiesel is a nearly perfect substitute for the standard diesel, and one can imagine several types of alternative energy (wind or solar energy) to be perfect substitutes for coal sooner or later. Hence, we can suppose that the more substitutes for non-renewable resources are found, the more flexible will be the overall production process at the macroeconomic level. Furthermore, this assumption allows our article to serve as a bridge to Tahvonen and Salo (2001), who assume perfect substitutability between non-renewable and renewable resources.

\subsection{CES and biased technical change}

As an alternative to a changing elasticity of substitution, we shall introduce technical change in the distribution parameter $\psi(t)$, to which we shall refer to as biased technical change (or factor-augmenting technical change).

By biased technical change, we shall mean changes in the productivity of one production factor relative to the other. This interpretation can be brought closer in line with intuition by transforming the CES form (1), which we assume throughout the paper, to a form where unit productivities are given explicitly. We have the following transformation in mind. ${ }^{6}$ Take

$$
R=B\left[\psi R_{N}^{\theta}+(1-\psi) R_{R}^{\theta}\right]^{\frac{1}{\theta}}
$$

which is Eq. (1), with a provisional addition of the factor-neutral productivity term $B>0$, and without time arguments, which have been omitted for convenience.

\footnotetext{
${ }^{6}$ For a formal justification that the transformed version of a CES function is also consistent with normalization, see Appendix A.1.
} 
Then, define $a=\psi^{\frac{1}{\theta}} B$ and $b=(1-\psi)^{\frac{1}{\theta}} B$, which implies $\psi=\frac{a^{\theta}}{a^{\theta}+b^{\theta}}$ and $B=\left(a^{\theta}+b^{\theta}\right)^{\frac{1}{\theta}}$. Transforming (4) yields the final result:

$$
\begin{aligned}
R & =\left(a^{\theta}+b^{\theta}\right)^{\frac{1}{\theta}}\left(\frac{a^{\theta}}{a^{\theta}+b^{\theta}} R_{N}^{\theta}+\frac{b^{\theta}}{a^{\theta}+b^{\theta}} R_{R}^{\theta}\right)^{\frac{1}{\theta}} \\
& =\left(\left(a R_{N}\right)^{\theta}+\left(b R_{R}\right)^{\theta}\right)^{\frac{1}{\theta}} .
\end{aligned}
$$

Thus, for each fixed substitution parameter $\theta \in(-\infty, 1), B$ is the common growth factor of both unit productivities ( $a$ and $b$ ), whereas the difference in their speed of growth is captured by $\psi$. In the remainder of the article, we shall set the factor-neutral growth parameter in equation (4) to $B=\left(a^{\theta}+b^{\theta}\right)^{\frac{1}{\theta}} \equiv 1$, which will allow us to focus on the effects induced by the difference in the speed of technical change between $R_{N}(t)$ and $R_{R}(t)$ only. As demonstrated, this difference can be captured by the distribution parameter. Setting $B=1$ is firstly required for the purpose of normalization, but one should think about this predominantly as a means of concentrating on the implications of the difference in the speeds of biased technical change. Thus, in different words, we scale the function in order to draw our main attention away from the level effect induced by $B$ to the actual biased effect induced by the differences in speeds. This shift in attention is the reason which allows us to draw further conclusions than the current literature. We still allow for factor-neutral technical change in the full model, but we must assume that it does not come from the intermediate input to avoid problems of normalization. ${ }^{7}$

The literature mainly focuses on efficiency improvements in the usage of the non-renewable resource. We are, however, going to focus on faster improvements in the usage of the renewable resource. Following this idea, we shall assume that technical change affects the relative share of the two inputs by changing the distribution factor, $\psi(t)$, as follows:

$$
\psi(t)=\psi(0) e^{z t}, \quad \psi(t) \in[0,1], \quad \psi(0) \in(0,1)
$$

where the growth rate of $\psi(t)$ is thus $z \leq 0$.

\footnotetext{
${ }^{7}$ These important issues of normalization have been neglected in the prior literature, but are clearly important if one does not want to compare inter-family CES functions. Obviously, the need for normalization in CES functions increases the complexity of the analysis to a substantial degree, as is visible in our case.
} 


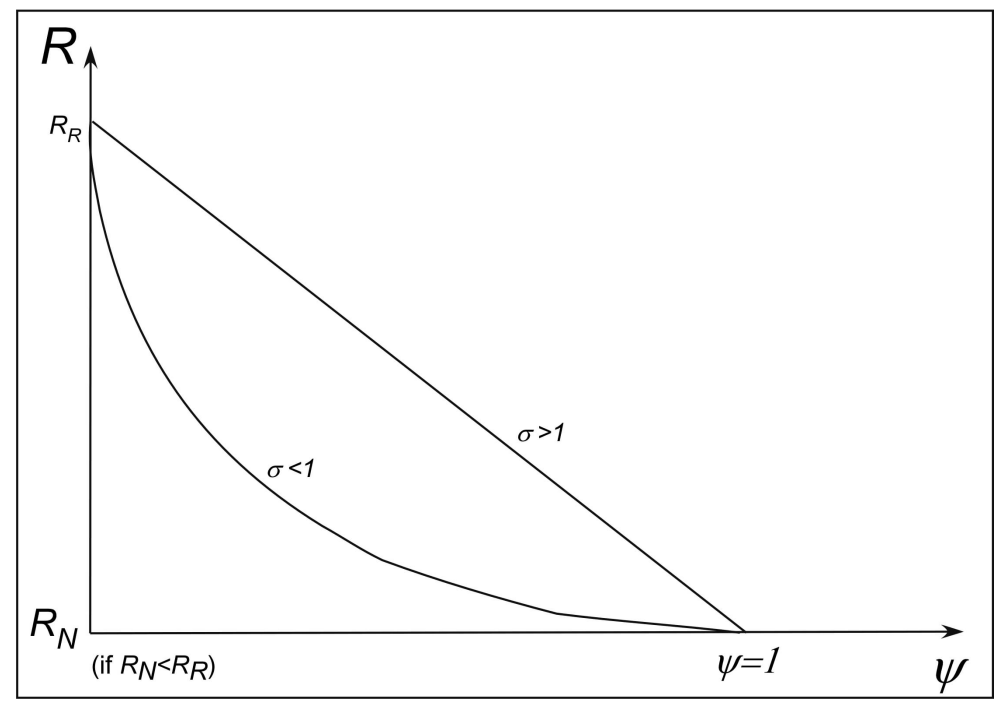

Figure 2: $R$ as a function of $\psi$

The effect of changing $\psi(t)$ on $R(t)$ is

$$
\frac{\partial R(t)}{\partial \psi(t)}=\frac{1}{\theta(t)}\left(R_{N}(t)^{\theta(t)}-R_{R}(t)^{\theta(t)}\right)\left[\psi(t) R_{N}(t)^{\theta(t)}+(1-\psi(t)) R_{R}(t)^{\theta(t)}\right]^{\frac{1-\theta(t)}{\theta(t)}} .
$$

The effect of $\psi(t)$ on $R(t)$ is less straightforward than the effect of $\sigma(t)$. We obtain that $\frac{\partial R(t)}{\partial \psi(t)}>0$ if $R_{N}>R_{R}$ and $\sigma>1$ or if $R_{N}<R_{R}$ and $\sigma<1$. If the signs are reversed then increases in $\psi(t)$ have a negative effect on $R(t)$. Figure 2 illustrates the effect of changing $\psi$ for the case of $R_{N}<R_{R}$. The lower the possibility to substitute the stronger the initial effect of changes in $\psi$. In the extreme, for Leontief inputs, a change from $\psi=0$ to $\psi>0$ will result in a reduction in the intermediate output from $R_{R}$ to $R_{N}$. This type of technical change can thus be viewed as varying the relative productivity of $R_{R}$ and $R_{N}$. The marginal rate of technical substitution is given by MRTS $=\frac{\psi}{1-\psi}\left(\frac{R_{N}}{R_{R}}\right)^{-\frac{1}{\sigma}}$. Changes in $\psi$ thus change the slope of the isoquant $R$. In section 3.3, we show several implications of assuming this kind of technical progress and provide some new suggestions for policy making. 


\subsection{Closing the model}

Taking the growth rate of $R(t)$ and dropping time subscripts yields

$$
\hat{R}=\epsilon_{N} \hat{R}_{N}+\epsilon_{R} \hat{R}_{R}+\epsilon_{\sigma} \hat{\sigma}+\epsilon_{\psi} \hat{\psi}
$$

where we assume that either $\hat{\psi}=0$ or $\hat{\sigma}=0$, and the partial elasticities, $\epsilon_{i}=\frac{\partial R}{\partial i} \frac{i}{R}$, for $i=R_{N}, R_{R}, \sigma, \psi$, are given by

$$
\begin{aligned}
\epsilon_{N} & =\frac{\psi R_{N}^{\theta}}{R^{\theta}} \\
\epsilon_{R} & =\frac{(1-\psi) R_{R}^{\theta}}{R^{\theta}}, \\
\epsilon_{\sigma} & =\frac{\psi R_{N}^{\theta} \ln R_{N}+(1-\psi) R_{R}^{\theta} \ln R_{R}-R^{\theta} \ln R}{(\sigma-1) R^{\theta}}, \\
\epsilon_{\psi} & =\frac{\psi\left(R_{N}^{\theta}-R_{R}^{\theta}\right)}{\theta R^{\theta}} .
\end{aligned}
$$

Both $\epsilon_{N} \in[0,1]$ and $\epsilon_{R} \in[0,1]$ function as shares in the traditional sense. This is due to the assumption of constant returns to scale in the CES function. Furthermore, the partial elasticity of $R$ with respect to $\sigma$ is always positive, implying that improvements in the elasticity of substitution always increase the output. We also notice that, given $\theta>(<) 0$, we have that $\epsilon_{\psi}>(<) 0$ if $R_{N}>R_{R}$ and $\epsilon_{\psi}<(>) 0$ if $R_{N}<R_{R}$, suggesting that technical change in the distribution factor should always go in the direction of the relatively abundant resource input if the resources are substitutes, and in the direction of the relatively scarce resource input if the resources are complements. This suggests that technical change in the distribution parameter is most useful when it goes to the resource that is most important for production.

Having analyzed the properties of the function describing $R(t)$, we shall now assume that $R(t)$ adds to production as an intermediate input in the following way:

$$
\begin{aligned}
& Y=F\left(A, K, L, R_{N}, R_{R}, \sigma, \psi\right)=A K^{\alpha} L^{1-\alpha-\beta} R^{\beta} \\
& \hat{Y}=\hat{A}+\alpha \hat{K}+(1-\alpha-\beta) \hat{L}+\beta\left[\epsilon_{N} \hat{R}_{N}+\epsilon_{R} \hat{R}_{R}+\epsilon_{\sigma} \hat{\sigma}+\epsilon_{\psi} \hat{\psi}\right]
\end{aligned}
$$

where $Y, A, K, L$ denote income, factor-neutral technical change, capital, and labor, respectively, and where we drop the time subscripts for convenience. An assumption 
behind this production function is that the intermediate good, the resource bundle, is essential for production, as $R=0$ implies $Y=0$. This specification clearly shows that our main concern lies with the way in which substitution and technical change in the nonrenewable versus renewable input affect overall production opportunities. It also shows that we decompose technical change into three different types, namely technical change through $\sigma(t)$, factor-neutral technical change through $A(t)$ and biased technical change through $\psi(t)$.

In order to understand how our extensions affect the properties of this nested production function in the long run, we shall assume the following laws of motion for the different variables:

$$
\begin{aligned}
& R_{R}(t) \equiv \text { constant } \\
& R_{N}(t)=R_{N}(0) e^{x t}, \quad x<0, \\
& L(t)=L(0) e^{n t}, \quad n \geq 0, \\
& A(t)=A(0) e^{g t}, \quad g \geq 0,
\end{aligned}
$$

and select one of the two following alternatives:

$$
\left\{\begin{array} { l } 
{ \sigma ( t ) = \sigma ( 0 ) e ^ { s t } , \quad s \geq 0 } \\
{ \psi ( t ) \equiv \text { constant } , }
\end{array} \quad \text { or } \quad \left\{\begin{array}{l}
\sigma(t) \equiv \text { constant }, \\
\psi(t)=\psi(0) e^{z t}, \quad z \leq 0 .
\end{array}\right.\right.
$$

We admit that the assumption of a constant renewable resource flow (and thus, of a constant renewable resource stock as well), which we shall maintain throughout the paper, is quite strong. Moreover, looking at the function describing the evolution of the renewable resource stock, $\dot{S}_{R}(t)=\varpi\left(S_{R}(t)\right)-R_{R}(t)$, we see that our assumption inevitably implies $R_{R}(t)=\varpi\left(S_{R}(t)\right)$. In simpler words, we just assume that as many renewable resources are being used as re-grow each period, thus keeping the total amount constant.

There are several reasons why one could assume this. One way to justify this is by assuming that renewable resource extraction is carried out not by utility-maximizers, but by an independent entity that is concerned with the preservation of the renewable resource stock over the long run.

Another justification comes directly from Dasgupta and Heal's (1974) analysis: they consider the renewable resource to be "(...) a perfectly durable commodity which provides 
a flow of services at [a] constant rate (...)" (Dasgupta and Heal, 1974, p. 19). Whereas they are interested in the optimal decision process given the uncertain timing of the discovery of this renewable resource, we shall focus on the case where the discovery has already taken place.

Two recent articles that deal with optimization in both resource inputs are Tahvonen and Salo (2001) and André and Cerdá (2005). Whereas Tahvonen and Salo (2001) use perfect substitutability between the resource inputs, André and Cerdá (2005) are more general by allowing $\sigma \in[0, \infty)$. Optimizing subject to both resource inputs allows them to find a corner solution where it might be useful to delay the exploitation of the renewable resource in order to allow for its stock to grow towards the maximum sustainable yield. Such result is not possible here, as we concentrate on interior solutions only, with constant renewable resource flows. However, we see their results as complementary to the results obtained by our approach.

We shall now commence to describe the asymptotic properties of the production function given the laws of motion that we assume. ${ }^{8}$

\section{Asymptotic results}

\subsection{Flexibility is the key}

We shall first quickly review the properties of the benchmark model, with results close to the established literature (see e.g. Dasgupta and Heal, 1974). Our benchmark assumptions are that there is no technical change in $\sigma$ and $\psi$, thus $s=z=0$. We can distinguish two important cases, one in which $\sigma>1$, and one in which $\sigma \leq 1$.

If $\sigma>1$, and so the intermediate inputs are gross substitutes, then the following asymp-

\footnotetext{
${ }^{8}$ The effect of depletion of the non-renewable resource stock on the partial elasticities are given in Appendix A.2.
} 
totic results hold:

$$
\begin{array}{r}
Y=\left.F\left(A, K, L, R_{N}, R_{R}\right)\right|_{R_{N}=0}>0 \\
\lim _{t \rightarrow \infty} \epsilon_{N}=0 \quad \lim _{t \rightarrow \infty} \epsilon_{R}=1 \\
\Rightarrow \quad \lim _{t \rightarrow \infty} \hat{y}=\lim _{t \rightarrow \infty}(\hat{Y}-n)=g+\alpha \hat{K}-(\alpha+\beta) n .
\end{array}
$$

As the non-renewable resource is not essential for production, it becomes a virtually negligible input once it tends to zero. Thus per capita income growth can stay positive if population growth is not too fast.

On the contrary, if $\sigma \leq 1$ then the non-renewable resource is essential for production as

$$
\begin{array}{r}
Y=\left.F\left(A, K, L, R_{N}, R_{R}\right)\right|_{R_{N}=0}=0 \\
\lim _{t \rightarrow \infty} \epsilon_{N}=1 \quad \lim _{t \rightarrow \infty} \epsilon_{R}=0 \\
\Rightarrow \quad \lim _{t \rightarrow \infty}(\hat{Y}-n)=g+\alpha \hat{K}-(\alpha+\beta) n+\beta x .
\end{array}
$$

The partial elasticity of the non-renewable resource tends to one because if the inputs are gross complements, then $R_{N}$ drives the output if it approaches zero. In this case both the rate of population growth and the rate of resource depletion, $x<0$, provide a drag on per capita income.

This benchmark case thus confirms the importance of flexibility in the production process, a result that carries forward from the classical literature.

\subsection{Increasing flexibility saves the day}

We shall now analyze the effect of allowing for technical change in the elasticity of substitution, but not in the distribution parameter, such that $s>0, z=0$. The long-run 
asymptotic result is straightforward and given by

$$
\begin{array}{r}
Y=\left.F\left(A, K, L, R_{N}, R_{R}\right)\right|_{R_{N}=0}>0 \\
\lim _{t \rightarrow \infty} \sigma=\infty \Rightarrow \lim _{t \rightarrow \infty} \theta=1 \\
\lim _{t \rightarrow \infty} \epsilon_{N}=0 \quad \lim _{t \rightarrow \infty} \epsilon_{R}=1 \quad \lim _{t \rightarrow \infty} \epsilon_{\sigma}=0 \\
\Rightarrow \quad \lim _{t \rightarrow \infty}(\hat{Y}-n)=g+\alpha \hat{K}-(\alpha+\beta) n
\end{array}
$$

There is actually no need to assume unbounded growth in the elasticity of substitution. We would get the same asymptotic growth rate for income if we assumed that $\sigma(t)$ grows until some time $t_{0}$, after which $\sigma(t)>1$, and the non-renewable resource is not essential for production any more. Hence, the asymptotic properties hold as long as the elasticity of substitution manages to cross the "magical" barrier of one.

\subsection{Biased technical change}

In this part we abstract from technical change in the elasticity of substition, $s=0$, and concentrate on the effects of biased technical change, with $z<0$. We thus assume that technical change improves the efficiency of the renewable resource more quickly than it improves the efficiency of the non-renewable resource. A priori, it may seem counterintuitive to suggest this as a way out of the long-run constraint imposed by the essentiality of a non-renewable resource. We shall however show and explain why and when this kind of biased technical change is useful. Here we are dealing with three cases, $\sigma>1$ and $\sigma<1$, and with the Cobb-Douglas as a special case.

If we have high substitutability of the intermediate inputs $(\sigma>1)$, then the following asymptotic properties hold:

$$
\begin{array}{r}
Y=\left.F\left(A, K, L, R_{N}, R_{R}\right)\right|_{R_{N}=0}>0 \\
\lim _{t \rightarrow \infty} \epsilon_{N}=0 \quad \lim _{t \rightarrow \infty} \epsilon_{R}=1 \quad \lim _{t \rightarrow \infty} \psi=0 \\
\Rightarrow \quad \lim _{t \rightarrow \infty}(\hat{Y}-n)=g+\alpha \hat{K}-(\alpha+\beta) n .
\end{array}
$$

As the renewable resource can be easily substituted for the non-renewable resource, its flow into production of the intermediate good will be decisive for output when then stock 
of the non-renewable resource is close to depletion. In this case, biased technical change has no effect on the asymptotic results.

Things look vastly different for $0<\sigma<1(\theta<0)$ though. Here the speed of technical change is crucial for the long-run results.

Case $1(z+\theta x>0)$ This is the case of slow technical change. The asymptotic results in this case are given by

$$
\begin{array}{r}
Y=\left.F\left(A, K, L, R_{N}, R_{R}\right)\right|_{R_{N}=0}=0 \\
\lim _{t \rightarrow \infty} \epsilon_{N}=1 \quad \lim _{t \rightarrow \infty} \epsilon_{R}=0 \\
\Rightarrow \lim _{t \rightarrow \infty}(\hat{Y}-n)=g+\alpha \hat{K}-(\alpha+\beta) n+\beta\left(x+\frac{z}{\theta}\right) .
\end{array}
$$

If the relative productivity of the renewable resource increases too slowly compared to the rate of depletion of the non-renewable resource stock (weighted by the elasticity of substitution), then the renewable resource is not able to fully compensate for the reduction in the intermediate good due to the shrinking flow of the non-renewable resource. Additionally, the greater the degree of complementarity between the resource inputs, the faster must be the increase in the relative productivity of the renewable resource.

Case $2(z+\theta x \leq 0)$ This assumption implies that the technical change is quick enough to fully compensate for the declining flow of non-renewable resources. This condition is more likely to be satisfied the weaker the complementarity of resource inputs.

Case 3 (Cobb-Douglas) For the Cobb-Douglas case, where $\sigma=1$, one would a priori expect a result along the lines of $\sigma<1$, the case of complementarity. However, the same asymptotic properties hold as in the case where $\sigma>1$.

These results, together with the analysis of equation (7), allow us to draw conclusions which are a little more precise than those of recent research. For example, André and Cerdá (2005) derive an equation describing the optimal dynamic evolution of the resource input ratio (their equation 6) and the optimal output path (equation 8 ), and then conclude from these that "equations (6) and (8) express, in a mathematical way, the interest (and, in the long run, the need) to promote the research and use of renewable energy sources (...) to substitute nonrenewable energies (...) from a sustainability perspective." Our analysis allows to find that biased technical change has its merits when it is directed to the most important resource input. This might imply that in the short run it would be 
optimal to invest more in research promoting the non-renewable resource rather than the renewable one.

In the long run, however, the result by André and Cerdá (2005) prevails. Under high substitutability $(\sigma \geq 1)$, production is driven by the relatively abundant renewable resource. It is therefore useful to improve the productivity of this resource by more than the productivity of the non-renewable resource. Under complementarity $(\sigma<1)$, it is the non-renewable resource that is more important in the long run, as it drives production due to its decreasing input flow. In this case, only a quick improvement in the relative productivity of the renewable resource can compensate for the reduction in the non-renewable resource flows. ${ }^{9}$

\section{The Hotelling model revisited}

\subsection{The model}

We shall now extend our insights gained from the asymptotic properties of the production function by using this function in an optimal growth model. The questions we attempt to answer hereby are whether our asymptotic results carry forward to an optimal growth model, and especially how the model behaves in the short to medium run. We shall use a Ramsey-type model with an infinite planning horizon, where a representative agent maximizes discounted utility subject to an equation of motion of the non-renewable resource stock, and the production function introduced in section 2 .

\footnotetext{
${ }^{9}$ These results should be verified in an optimal growth framework which fully endogenizes research in both resource inputs.
} 
Formally, this means that the infinitely-lived representative agent maximizes

$$
\begin{aligned}
\max _{R_{N}(t)} \int_{0}^{\infty} U(Y(t)) e^{-\rho t} d t & \text { subject to } \\
\dot{S}_{N}(t) & =-R_{N}(t), \\
R_{R}(t) & \equiv \text { const, } \\
Y(t) & =F\left(A, L, R_{N}, R_{R}\right)=A(t) L(t)^{1-\beta} R(t)^{\beta}, \\
R(t) & =\left[\psi(t) R_{N}(t)^{\theta(t)}+(1-\psi(t)) R_{R}(t)^{\theta(t)}\right]^{\frac{1}{\theta(t)}}
\end{aligned}
$$

Here we assume that $U(Y(t))=\frac{Y(t)^{1-\gamma}}{1-\gamma}$, where $\gamma=\frac{-Y U^{\prime \prime}(Y)}{U^{\prime}(Y)} \in(0, \infty)^{10}$ is the inverse of the intertemporal elasticity of substitution. We abstract from population growth by assuming that $L \equiv 1$ (in order to obtain clear short-term results). In addition, we carry all our previous assumptions forward. Namely, we again decompose technical change into three components: $A(t)=A(0) e^{g t}$ with $g \geq 0, \sigma(t)=\sigma(0) e^{s t}$ with $s \geq 0, \psi(t)=\psi(0) e^{z t}$ with $z \leq 0$. On top of this, we assume that either $s=0$ or $z=0$. In contrast to the previous section, we now also abstract from capital accumulation.

The first-order conditions give us (see Appendix A.3 for more details):

$$
\left(\frac{\widehat{\partial U(Y)}}{\partial R_{N}}\right)=\rho
$$

This is the Ramsey-Hotelling condition that characterizes the interior solution of the optimization problem. Rewriting, we obtain that

$$
\hat{Y}=\frac{\hat{F}_{N}-\rho}{\gamma}
$$

where $\hat{F}_{N}=\partial F / \partial R_{N}$, which states that the growth rate of income, $\hat{Y}$, is positive if the growth rate of the marginal product of the non-renewable resource, $\hat{F}_{N}$, exceeds the discount rate.

This result is isomorphic to the outcome of the "cake-eating" model (see e.g. Dasgupta and Heal, 1974). In the cake-eating model, the marginal product of the non-renewable resource is only a function of the rate of depletion and factor-neutral technical change. Hence, without large enough factor-neutral technical change, the growth rate of the marginal

\footnotetext{
${ }^{10} \gamma>1$ implies that utility is bounded from above.
} 
product of the non-renewable resource will be negative for all times and income will decline continuously. Income growth can only be positive in case technical change is fast enough to keep the growth rate of the marginal product of the non-renewable resource above the discount rate. However, as we decompose technical change into three different kinds, we have more possibilities to achieve sustainable production. The way in which the model's results are changed due to the introduction of technical change in the elasticity of substitution and biased technical change through the distribution parameter will become clear in the subsequent subsections.

Solving equation (12) for $\hat{R}_{N}$, we obtain the optimal growth rate of the non-renewable resource extraction in terms of the other variables:

$$
\hat{R}_{N}=\frac{(1-\gamma) g-\rho+\left[((1-\gamma) \beta-\theta) \epsilon_{\sigma}-\frac{1}{\sigma}\left(\ln R-\ln R_{N}\right)\right] s+\left[((1-\gamma) \beta-\theta) \epsilon_{\psi}+1\right] z}{(1-\theta)-((1-\gamma) \beta-\theta) \epsilon_{N}} .
$$

As is proved in Appendix A.4, the denominator of the above expression is always positive. We also notice that all three kinds of technical change, factor-neutral technical change $(g>0)$, the technical change in the elasticity of substitution $(s>0)$, as well as biased technical change $(z<0)$, affect the optimal growth rate of $R_{N}$.

\subsection{Comparative statics}

We shall now turn to comparative statics to provide further insights into the effects which some parameters or variables exert on the growth rate of income. On the optimal growth path we obtain the following comparative statics:

$$
\begin{aligned}
& \frac{\partial \hat{Y}}{\partial \rho}=-\frac{\beta \epsilon_{N}}{(1-\theta)-((1-\gamma) \beta-\theta) \epsilon_{N}}<0, \\
& \frac{\partial \hat{Y}}{\partial g}=\frac{(1-\theta)+\theta \epsilon_{N}}{(1-\theta)-((1-\gamma) \beta-\theta) \epsilon_{N}}>0, \\
& \frac{\partial \hat{Y}}{\partial s}=\frac{\beta(1-\theta)\left[\epsilon_{\sigma}-\epsilon_{N}\left(\ln R-\ln R_{N}\right)\right]}{(1-\theta)-((1-\gamma) \beta-\theta) \epsilon_{N}}, \\
& \frac{\partial \hat{Y}}{\partial z}=\frac{\beta\left[\epsilon_{N}+\epsilon_{\psi}(1-\theta)\right]}{(1-\theta)-((1-\gamma) \beta-\theta) \epsilon_{N}} .
\end{aligned}
$$

We find that, along the optimal path, the discount rate affects the growth rate of income always negatively. Moreover, the less we care about the future (the higher $\rho$ ), the stronger 
is its negative impact on income growth (Eq. (14)). Clearly, the less we care about the future the more resources we use now, resulting in a larger current level of income with less growth potential or, better, with less resources to use later and thus a stronger drag on economic growth.

As expected, the growth rate of income is unambiguously positively related to the factorneutral technical change (Eq. (15)). This result also carries forward from the standard literature.

We know from section 2 that changes in the distribution factor should always go towards the kind of resource which is more important for production. Equation (17) suggests, in a similar way, that increases in $z$ have a positive effect of income growth if $\epsilon_{\psi}>-\frac{\epsilon_{N}}{1-\theta}(<0)$. Hence, a sufficient condition for a positive effect of $z$ on income growth is $\epsilon_{\psi}>0$, i.e. if the relatively more important resource is subject to faster technical change than the other resource input.

The effect of $s$ on income growth depends on whether more renewable or more nonrenewable inputs are used in production. If more non-renewable inputs are used then the effect of $s$ on income growth is positive. However, if more renewable resources are used (which is inevitably the case because the non-renewable resources are gradually depleted), then the effect of $s$ on income growth depends on the importance of the non-renewable resource for production relative to the partial elasticity of $\sigma$ and cannot be unambiguously signed.

\subsection{Simulations and analysis}

As it is not possible to solve the differential equation (13) analytically in the general case, we shall use numerical simulations in order to provide more understanding to the short- and medium-run dynamic paths resulting from the optimizing behavior of the representative agent ${ }^{11}$. We will additionally compute the limit value of the extraction

\footnotetext{
${ }^{11}$ We use the following parameter choices for the simulations: $\beta=1 / 3, \gamma=2, R_{R}=2, \rho=0.05$, $\sigma(0)=0.1, \psi(0)=0.9, S(0)=300$. The simulations are run using the Matlab routine ODE45. They are done in two steps. In the first step, we implement the "shooting" method to find the initial amount of extracted resource $R_{N}(0)$, i.e. we run the routine for a range of initial values and pick the one that guarantees $S_{N}(t) \rightarrow 0$. Since of course, the simulations have to be stopped in finite time, we approximate
} 
rate of the non-renewable resource, which helps understand under which circumstances we can expect exhaustion of the non-renewable resource stock in finite time, and under which in infinite time.

We shall provide the limits of the optimal rate of extraction for our benchmark case, the flexibility case, and the biased technical change case, along with the simulation results.

In the benchmark case, where $s=0$ and $z=0$, we derive the following limits:

$$
\lim _{t \rightarrow \infty} \hat{R}_{N}= \begin{cases}\frac{(1-\gamma) g-\rho}{1-\theta} & \sigma>1 \\ \frac{(1-\gamma) g-\rho}{1-(1-\gamma) \beta \psi} & \sigma=1 \\ \frac{(1-\gamma) g-\rho}{1-(1-\gamma) \beta} & \sigma<1\end{cases}
$$

These limits promise an interior solution without depletion in finite time only if $(1-\gamma) g-$ $\rho<0$. The more patient the representative agent, i.e. the smaller is $\rho$, the more important is the future for her and therefore the larger the growth rate of resource extraction. If we assume away the possibility of depletion in finite time, then this implies that initially smaller amounts of resources will be utilized. Furthermore, depletion in finite time is possible only if utility is unbounded $(\gamma<1)$. This result holds irrespectively of whether the resource inputs are gross substitutes or gross complements.

In addition, the larger is $\gamma$ the smaller is the willingness for inter-temporal substitution of income. This implies that for larger $\gamma$, more resources will be used by the agent initially, and therefore the growth rate of resource extraction must be lower in order for the agent to have some resources left for future consumption.

From now on, we shall rule out finite-time depletion, and thus guarantee an interior solution, by assuming $(1-\gamma) g+z-\rho<0$.

In order to show the optimal path of income in this benchmark case, we resort to simulations, given in Figure 3.

The interpretation of the above result is as follows. The discounted utilitarian criterion regards future consumption as less important than current consumption, wherefore most this limit value by $S_{N}(300)$. This approximation is valid, because with our parameter choices, the remaining stock of non-renewable resource becomes negligible by the time $t=300$. In the second step, we re-run the routine with the correct value of $R_{N}(0)$ as an initial condition. Having obtained the time path of $R_{N}$, we calculate the time paths of other variables by straightforward inserting. 

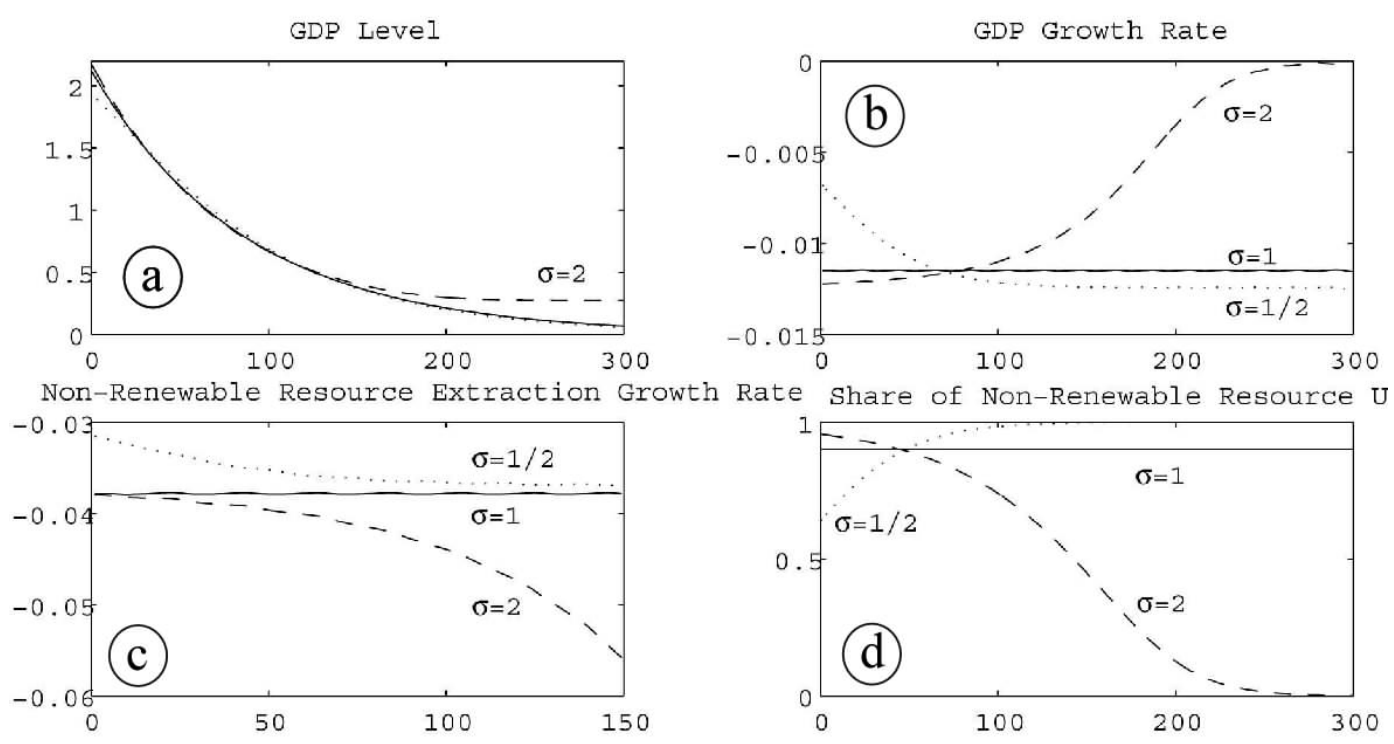

Figure 3: Benchmark case, $s=0, z=0$

non-renewable resources will be extracted when utility seems most valuable. The simulations show that in case the non-renewable resource is not essential for production $(\sigma=2)$, the share of $R_{N}$ is initially very large, suggesting that most non-renewable resources are used initially, and then declines to zero over time (d). Thus the level of GDP is initially larger for the case of high substitutability in comparison to the case of $\sigma \leq 1$, as technical efficiency (as measured by $\sigma$ ) does not constrain the intermediate input $R(t)$ by complementarity problems (a). The GDP growth rate is initially higher in the case of complementarity as at first more non-renewable resources are conserved as production is depending on those resources later (b). Moreover, the amount of the non-renewable resource used in production is divided more equally over time the lower is $\sigma$ due to its essentiality for production ${ }^{12}$ (c). Nevertheless though, if the non-renewable resource and the renewable one are gross complements in production, the decrease in the amount of non-renewable resources available decreases the overall resource bundle that can finally be used in production. In such case, the non-renewable resource is driving the size of the resource bundle $R(t)$, implying that the share of the non-renewable resource in the

\footnotetext{
${ }^{12}$ The dashed line representing the growth rate of extraction for the case of $\sigma=2$ tends to its minimum at -0.1 , which is outside of the graph.
} 
resource bundle will go to one $(\mathrm{d})$. Hence, the (negative) GDP growth rate will become smaller and smaller (b). In contrast to this, if the non-renewable resource is inessential $(\sigma>1)$ then, as less and less of it remains available, its share in the resource bundle will drop to zero (d). In this sense, the non-renewable resource allows greater GDP levels as long as it is available, but as soon as it gets depleted, GDP goes to the level that it would have had, had it been produced without the non-renewable resource.

Moving on to the flexibility case, where we assume $s>0$ and $z=0$, we know that from a certain time $t_{0} \geq 0$ onwards, the intermediate inputs will be gross substitutes as after this point in time the elasticity of substitution will exceed one. In the limit, the extraction growth rate approaches minus infinity:

$$
\lim _{t \rightarrow \infty} \hat{R}_{N}=-\infty
$$

This result is intuitive, because perfect substitutability between non-renewable and renewable resources allows us to utilize the non-renewable resource without compromising the renewable one in any way (in comparison to the case of $\sigma \leq 1$, for example).
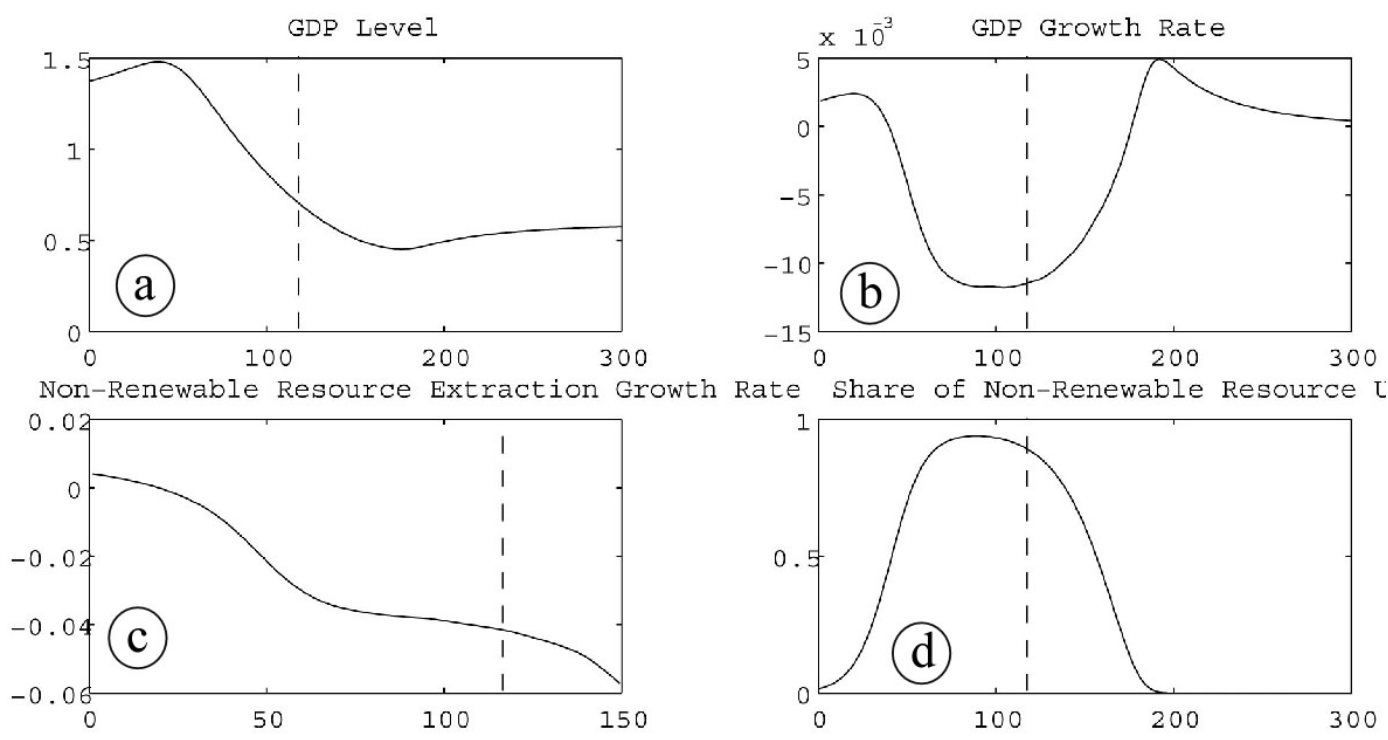

Figure 4: Flexibility case, $s>0, z=0$

The simulations ${ }^{13}$ allow us to be more precise with respect to the short- to medium-run

\footnotetext{
${ }^{13}$ The vertical dashed lines show when the elasticity of substitution crosses one.
} 
results. As the elasticity of substitution increases it is optimal to, at first, utilize nonrenewable resources at an increasing rate (c). This is because, initially, the improvements in technical efficiency allow for large increases in the intermediate input $R(t)^{14}$. Hence, this allows for an increasing growth rate of income (b). However, these initial exponential increases in $R(t)$, due to the increasing elasticity of substitution, level off quickly, such that consecutive improvements in $\sigma(t)$ increase the overall resource bundle only slightly. It is now optimal to slow down the extraction of the non-renewable resource, as it is still essential for production. At this time, the model is rather similar to the benchmark case with an essential resource and produces a negative growth rate of income. At one point in time, the technical efficiency will have improved substantially enough, so that the non-renewable resource will not be essential for production any more. The model then traces the benchmark case again, but with a non-essential non-renewable resource (d). However, continued improvements in the technical efficiency parameter result in further improvements in the intermediate good production process. Therefore, the economic growth rate can be positive (b). As the consecutive improvements in efficiency increase the intermediate resource by less and less, and as the non-renewable resource stock is depleted, the growth rate of income tends to zero from above.

Generally speaking, one can notice that the changing elasticity of substitution pulls double-duty, as suggested in section 2. On the one hand, it reflects technical efficiency, and on the other hand, it reflects the essentiality of the resource.

The last case we challenge is the distribution case, where we assume that there is no technical change in the elasticity of substitution, but there is biased technical change in the distribution parameter $(z<0)$.

If $s=0$ and $z<0$, then the limit extraction rate of the non-renewable resource depends

\footnotetext{
${ }^{14}$ The initial level of $\sigma(t)$ is set at $\sigma(0)=0.1$, which corresponds to the strongly increasing part of Figure 1.
} 
on the values of various parameters:

$$
\lim _{t \rightarrow \infty} \hat{R}_{N}= \begin{cases}\frac{(1-\gamma) g-\rho+\frac{((1-\gamma) \beta) z}{\theta},}{1-\theta}, & \sigma<1, \\ \frac{(1-\gamma) g-\rho-((1-\gamma) \beta-\theta) \frac{z}{\theta(1-\psi)}}{1-\theta}, & \sigma>1, \\ -\infty, & \sigma=1, \gamma>1, \\ z-\rho<0, & \sigma=1, \gamma=1, \\ +\infty, & \sigma=1, \gamma<1 .\end{cases}
$$

Hence, we see that the interior solution is not always guaranteed, even if one keeps the assumption $(1-\gamma) g+z-\rho<0$ in mind. For instance, if $\sigma=1$ and $\gamma<1$, then biased technical change implies finite-time depletion of the non-renewable resource, whereas in the previous section, we learned that with $\sigma=1$ and biased technical change, sustainable production is possible. It turns out that the lure of unbounded utility $(\gamma<1)$ provides a barrier against such a chance.
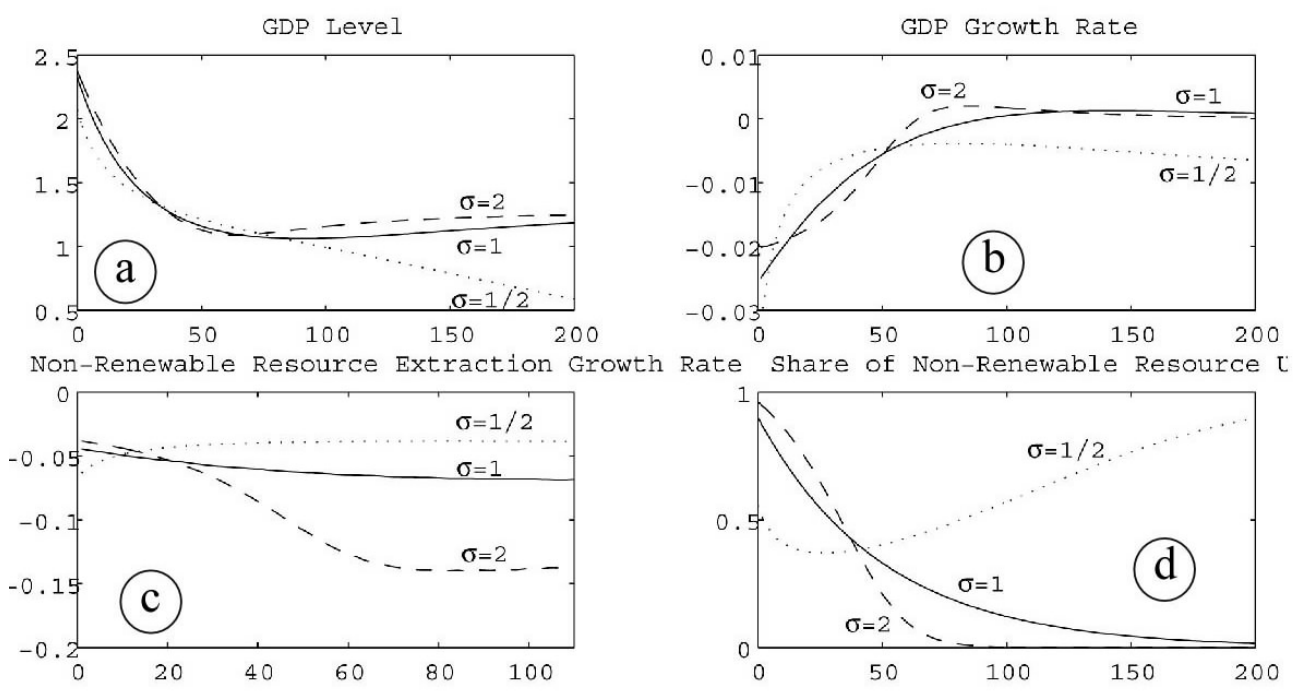

Figure 5: Distribution case, $s=0, z<0$

We can interpret the simulation results, presented in figure 5, as follows. The discounted utilitarian criterion leads the infinitely-lived agent to perceive current income as more 
important for the creation of his utility, so she initially utilizes most non-renewable resources (a). As in all previous cases, we observe the crucial role played by the elasticity of substitution. As suggested in section 2, if $\sigma<1$ then the relatively scarce resource drives production. Conversely, if $\sigma>1$, then the relatively more abundant resource will be decisive for the amount of the intermediate good. The numerical simulations show exactly this effect (d). Initially, more non-renewable resources are utilized in production. For the case of $\sigma<1$, this implies that the renewable resource constrains the amount of intermediate output, and therefore relatively quicker improvements in the marginal product of the renewable resource (as captured by $z<0$ ) reduce the drag on intermediate good output imposed by the renewable resource (b). In contrast to this, if the non-renewable resource is the largest input in the intermediate good and $\sigma>1$, then the non-renewable resource drives the intermediate good output. Hence, if the importance of the renewable resource now increases relatively to the non-renewable resource, then the effect on GDP growth is negative (b). These initial effects are reversed once the relative importance of the resource inputs changes due to the depletion of the non-renewable resource stock.

Further simulations show that in case the resources are gross complements, the speed of $z$ is vital in order to allow for a non-decreasing growth rate of income. For the case of $\sigma=1 / 2$, our simulations demonstrate that the assumed speed of biased technical change, $z=-0.02$, is not sufficient to outweigh the decrease in the non-renewable resource flow. Hence, the GDP level will tend to zero (a), a result in line with the benchmark case. However, if the speed of $z$ outweighs the reduction in the flow of non-renewable resources, then a positive, constant GDP level can be attainable. The speed of $z$ must be such that the renewable resource becomes more and more important to production quick enough to compensate for the decreasing quantities of the essential non-renewable resource available.

\section{Conclusion}

In a world where production depends on limited and non-renewable inputs, the desire to extend our current consumption opportunities to future generations requires that we resort to alternative inputs which are able to sustain the production process at similar or even higher levels. This is not always possible, especially if renewable resource inputs 
have to be used complementarily to the non-renewable inputs. Various kinds of technical change have been viewed as a way out, as they help to utilize given resource inputs more efficiently.

This article investigates a new kind of technical change. We assume that technical change improves the substitutability between two inputs, in our case non-renewable and renewable resources. This approach follows recent suggestions in the literature on technical change and resources. For example, Bretschger (2005, p.150) suggests that "all possibilities of substitution and, specifically, the effects technology exerts on promoting substitution have to be studied." We furthermore provide reasons for which technical change in the elasticity of substitution can be viewed as improvements in technical efficiency. We first analyze the implications of technical change in the elasticity of substitution for the asymptotic growth rates and we then develop a Ramsey-Hotelling model in order to understand the short- to medium-run dynamics as well.

In the long run, these improvements in the substitutability will make non-renewable resources inessential for production and allow for improvements in the growth rate of income. This result is clearly intuitive. The classical literature already notices that a high enough elasticity of substitution renders an input inessential. However, the Ramsey-Hotelling model provides new insights to the effects of a changing elasticity. We show that the infinitely-lived agent will take the changes in the elasticity of substitution into account when choosing the optimal income growth path. In the short run, numerical simulations show that improvements in the elasticity of substitution can at least initially alleviate the drag which non-renewable resources exert on income. This is because the first increases in the elasticity have the largest impact on the overall resource bundle. However, in the medium run, depletion of the non-renewable resource is still able to drive growth and thus reduce the income level. Then, after the improvements in the elasticity of substitution turn the non-renewable resource inessential $(\sigma>1)$, the economy can return to a steady state with positive income and without non-renewable resources. Hence, the improvements in the elasticity of substitution have two effects. One, they improve upon the technical efficiency between the resource inputs. Two, they help rendering the non-renewable resource inessential for production.

In addition to analyzing technical change in the elasticity of substitution, we allow for biased technical change. This means that both resource inputs are subject to technical 
change, but that the productivity of one of the resources, in our case the renewable one, improves quicker than the other resource. Using this simplified approach, we notice that technical change is most useful when it is directed to the most important resource for production, i.e. when it is directed to the resource which is most decisive for the production level. Using a Ramsey-Hotelling model we show that these asymptotic results carry forward to the short-run results. Biased technical change is either most useful when it is directed to the most important resource, or when it is quick enough to compensate for reductions in the input which has slower technical change.

In the light of these findings, consecutive research should address the following points. Firstly, exogenous technical change in the substitutability should be endogenized. It is one of the most important, if not the most important, parameter in models with nonrenewable resources. This is by no means a simple task and valuable results might only be possible for special cases. Secondly, one should attempt to put more emphasis on endogenized biased technical change in resource inputs. Results will help deepening our understanding as to which kind of technical change should be promoted by policy makers. Thirdly and finally, we are in need of empirical evidence with respect to the substitutability of resources in production. The fact that we do not have a clue about their substitutability, even though the knowledge on this will be extremely helpful for policy making, is a true disappointment. 


\section{A Appendix}

\section{A.1 Normalization of the CES function family}

When assuming (1), and allowing for technical change in $\sigma(t)$ or $\psi(t)$, we simultaneously make a number of implicit assumptions. The assumption of a changing $\theta$ but a constant distribution parameter $\psi$ alone does not yield a single CES function, but a family of production functions, indexed by the elasticity parameter $\theta$ (or alternatively, by the elasticity of substitution $\sigma$ ). As time elapses, the value of this index changes. This causes one to jump between different members of the CES family. The same reasoning applies when we keep $\sigma$ constant and allow $\psi$ to change over time.

The elasticity parameter describes the curvature of a CES function, given its slope, and the distribution parameter describes the slope, given the curvature. It was graphical reasoning that brought de La Grandville (1989) to suggest to normalize CES functions: each family of these functions is characterized by a single point of tangency, functions that do not share this point of tangency do not belong to the family. The reason for normalization is that when one wants to isolate the effect of a certain parameter dimension on the value of a function, one needs to hold other dimensions constant. The parameters that allow for heterogeneity are for us in one case the elasticity of substitution and in the other case the distribution parameter $\psi$. As Klump and Preissler (2000) suggest, we have to normalize CES functions, because we do not want to confuse intra-family differences with inter-family ones.

In the following paragraphs, we shall prove that the approach we adopt in this article is indeed consistent with normalization.

Following Klump and Preissler (2000), we shall characterize a given normalized CES function family by a triple $\left(\mu_{0}, n_{0}, r_{0}\right)$, according to:

$$
\begin{aligned}
& \mu_{0}=\left(\frac{\frac{\partial R}{\partial R_{R}}}{\frac{\partial R}{\partial R_{N}}}\right)_{0}=\frac{1-\psi}{\psi} n_{0}^{1-\theta}, \\
& r_{0}=\left(\psi n_{0}^{\theta}+(1-\psi)\right)^{\frac{1}{\theta}},
\end{aligned}
$$

where $n_{0}$ is interpreted as $R_{N 0} / R_{R 0}$, which is the ratio of two arbitrary baseline resource flow quantities (not necessarily the ratio of resource flows at time $t=0$, as notation may 
suggest); the same applies to $r_{0}=R_{0} / R_{R 0} . \mu_{0}$ is the baseline marginal rate of technical substitution.

The Arrow et al. (1961) CES specification which we assume in (1), confronted with these requirements for the case of a changing elasticity of substitution, yields

$$
\begin{aligned}
& \psi=\frac{n_{0}^{1-\theta}}{n_{0}^{1-\theta}+\mu_{0}}(\equiv \psi(\sigma)), \\
& 1=r_{0}\left(\frac{n_{0}^{1-\theta}+\mu_{0}}{n_{0}+\mu_{0}}\right)^{\frac{1}{\theta}} \quad(\equiv C(\sigma)),
\end{aligned}
$$

where the left-hand sides are constants and the right-hand sides are functions of $\sigma$. These two conditions imply $n_{0}=1, r_{0}=1$ (or equivalently, $R_{N 0}=R_{R 0}=R_{0}$ ), and $\mu_{0}=\frac{1-\psi}{\psi}$. Thus, our approach is equivalent to considering a CES family normalized at $n_{0}=1, r_{0}=1$, and $\mu_{0}=\frac{1-\psi}{\psi}$, where $\psi \in(0,1)$ is a given constant. Although our preferred values are admittedly arbitrary, we do not lose generality here: we would have to pick one single triple $\left(\mu_{0}, n_{0}, r_{0}\right)$ anyway. Our choice is motivated by convenience and the desire for greatest possible simplification of the mathematics involved.

To analyze the effects of biased technical change on production processes, the above normalization is not appropriate, since it assumes constancy of the distribution parameter $\psi$. Instead, we shall propose an alternative normalization, which fixes the elasticity parameter $\sigma$ and allows for heterogeneity in the baseline marginal rate of technical substitution $\mu_{0}$.

We shall now characterize a given normalized CES function family by a triple $\left(\theta, n_{0}, r_{0}\right)$, following (21) and (22). This time, it implies

$$
\begin{aligned}
\psi & =\frac{n_{0}^{1-\theta}}{n_{0}^{1-\theta}+\mu_{0}}\left(\equiv \psi\left(\mu_{0}\right)\right), \\
1 & =r_{0}\left(\frac{n_{0}^{1-\theta}+\mu_{0}}{n_{0}+\mu_{0}}\right)^{\frac{1}{\theta}} \quad\left(\equiv C\left(\mu_{0}\right)\right) .
\end{aligned}
$$

For the right-hand side of the second equation to be independent of $\mu_{0}$ for all $\theta \neq 0$, we have to assume $n_{0}=1$, which in turn implies $r_{0}=1$ (or equivalently, again $R_{N 0}=R_{R 0}=$ $R_{0}$ ). Hence, we obtain $\psi\left(\mu_{0}\right)=\frac{1}{1+\mu_{0}}$. Changes in $\mu_{0}$, which is now the only dimension that allows for heterogeneity, are equivalent to changes in $\psi$, which we manipulate directly in the main text. 
If one perceives biased technical change through the prism of Eq. (5), then our normalization procedure implies $a=\left(\frac{1}{1+\mu_{0}}\right)^{\frac{1}{\theta}}$ and $b=\left(\frac{\mu_{0}}{1+\mu_{0}}\right)^{\frac{1}{\theta}}$. Note that we assume the common productivity factor to be $a^{\theta}+b^{\theta}=r_{0}^{\theta} \equiv 1$. This means that we abstract from factorneutral technical progress: we fix this dimension in order to get viable results concerning the biased technical change dimension, as described by $\mu_{0}$, or alternatively, $\psi$.

\section{A.2 Asymptotic results for the partial elasticities}

For any given $\sigma$ and $\psi$, we obtain the following limit values:

$$
\begin{aligned}
\lim _{R_{N} \rightarrow 0} \epsilon_{N}= \begin{cases}0, & \sigma>1, \\
\psi, & \sigma=1, \\
1, & \sigma<1,\end{cases} \\
\lim _{R_{N} \rightarrow 0} \epsilon_{\psi}= \begin{cases}\frac{-\psi}{\theta(1-\psi)}<0, & \sigma>1, \\
-\infty, & \sigma=1, \\
\frac{1}{\theta}<0, & \sigma<1,\end{cases} \\
\lim _{R_{N} \rightarrow 0} \epsilon_{\sigma}= \begin{cases}-\frac{1}{\sigma} \ln (1-\psi)>0, & \sigma>1, \\
+\infty, & \sigma \leq 1 .\end{cases}
\end{aligned}
$$

\section{A.3 Optimization of the Hotelling model}

We can write the Hamiltonian as follows (omitting time subscripts):

$$
H\left(R_{N}, S_{N}, \lambda\right)=U\left(A\left[\psi R_{N}^{\theta}+(1-\psi) R_{R}^{\theta}\right]^{\frac{\beta}{\theta}}\right) e^{-\rho t}-\lambda R_{N} .
$$

The Pontryagin Maximum conditions are:

$$
\begin{gathered}
\frac{\partial H}{\partial R_{N}}=0 \Rightarrow \lambda=\frac{\partial U}{\partial R_{N}} e^{-\rho t}, \\
\frac{\partial H}{\partial S_{N}}=-\dot{\lambda} \Rightarrow-\dot{\lambda}=0 .
\end{gathered}
$$


Differentiating equation (27) with respect to time and substituting into equation (28) gives

$$
\hat{Y}=\frac{\hat{F}_{N}-\rho}{\gamma},
$$

which is equation (12) in the main text. The marginal product of the non-renewable resource $R_{N}$ is given by $F_{N}=A \beta \psi R_{N}^{\theta-1} R^{\beta-\theta}$. Its growth rate is given by

$$
\hat{F}_{N}=g+z+(\theta-1) \hat{R}_{N}+(\beta-\theta)\left(\epsilon_{N} \hat{R}_{N}+\epsilon_{\psi} z+\epsilon_{\sigma} s\right)+\frac{1}{\sigma}\left[\log \left(R_{N}\right)-\log (R)\right] s .
$$

As the growth rate of income is $\hat{Y}=g+\beta\left(\epsilon_{N} \hat{R}_{N}+\epsilon_{\psi} z+\epsilon_{\sigma} s\right)$, we can substitute these two growth terms into equation (29) to get

$$
\begin{array}{r}
(1-\gamma) g+\left[1+(\beta-\theta) \epsilon_{\psi}-\gamma \beta \epsilon_{\psi}\right] z+\left[(\beta-\theta) \epsilon_{\sigma}-\gamma \beta \epsilon_{\sigma}\right] s-\rho+\frac{1}{\sigma}\left[\log \left(R_{N}\right)-\log (R)\right] s \\
=\left[\gamma \beta \epsilon_{N}+1-\theta-(\beta-\theta) \epsilon_{N}\right] \hat{R}_{N} .
\end{array}
$$

Solving for $\hat{R}_{N}$ gives us the optimal growth rate of the non-renewable resource extraction, equation (13).

\section{A.4 Proof that the denominator of $\hat{R}_{N}$ is always positive}

The denominator of $\hat{R}_{N}$ is given by $(1-\theta)-((1-\gamma) \beta-\theta) \epsilon_{N}$. Rewriting this gives $(1-\theta)-(1-\gamma) \beta \epsilon_{N}+\theta \epsilon_{N}+\epsilon_{N}-\epsilon_{N}=\left(1-\epsilon_{N}\right)(1-\theta)-[(1-\gamma) \beta-1] \epsilon_{N}$. As we know that $\epsilon_{N} \in[0,1]$ and $\theta \in(-\infty, 1]$, we also know that the first term is greater or equal to zero. As $\gamma \in(0, \infty), 0<\beta<1$, then $(1-\gamma) \beta-1<0$, so the denominator turns out to be a sum of two non-negative expressions, one of them being strictly positive. This implies that $(1-\theta)-((1-\gamma) \beta-\theta) \epsilon_{N}>0$. 


\section{References}

1. André, F. \& Cerdá, E. (2004) "On natural resource substitution", Economic Working Papers at Centro de Estudios Andaluces E2004/48, Centro de Estudios Andaluces, forthcoming in Resources Policy.

2. Arrow, K. \& Chenery, H. \& Minhas, B. \& Solow, R. (1961) "Capital-Labor Substitution and Economic Efficiency", Review of Economics and Statistics 43, pp.225-50.

3. Bretschger, L. (2005) "Economics of Technological Change and the Natural Environment: How Effective are Innovations as a Remedy for Resource Scarcity?", Ecological Economics 54 (2-3), pp.148-163 .

4. Dasgupta, P. \& Heal, G. (1974) "The Optimal Depletion of Exhaustible Resources", The Review of Economic Studies 41 (Symposium on the Economics of Exhaustible Resources), pp.3-28.

5. de La Grandville, O. (1989) "In Quest of the Slutsky Diamond", American Economic Review 79, pp.468-481.

6. Cleveland, C. \& Ruth, M. (1997) "When, Where, and By How Much Do Biophysical Limits Constrain the Economic Process? The Contribution of Nicholas GeorgescuRoegen to Ecological Economics", Ecological Economics 22, pp.203-223.

7. Grimaud, A. \& Rouge, L. (2005) "Polluting non-renewable resources, innovation and growth: welfare and environmental policy", Resource and Energy Economics 27(2), pp.109-129.

8. Groth, C. (2004) "Strictly Endogenous Growth with Non-renewable Resources Implies an Unbounded Growth Rate", Topics in Macroeconomics, Berkeley Electronic Press, vol. 4, article 1.

9. Groth, C. \& Schou, P. (2002) "Can non-renewable resources alleviate the knife-edge character of endogenous growth?", Oxford Economic Papers 54(3), pp.386-411.

10. Hicks, J. (1932) The Theory of Wages, $2^{\text {nd }}$ edition, London, Macmillan, 1963. 
11. Klump, R. \& Preissler, H. (2000) "CES Production Functions and Economic Growth", Scandinavian Journal of Economics 102, pp.41-56.

12. Romer, P. (1990) "Endogenous Technological Change", Journal of Political Economy 98 , pp. 71-102.

13. Scholz, C. \& Ziemes, G. (1999) "Exhaustible Resources, Monopolistic Competition, and Endogenous Growth", Environmental and Resource Economics 13, pp.169-185.

14. Schou, P. (2000) "Polluting Non-Renewable Resources and Growth", Environmental and Resource Economics 16, pp. 211-227.

15. Smulders, S. (1995) "Environmental Policy and Sustainable Economic Growth", De Economist 2, pp. 163-195

16. Solow, R. (1956) "A Contribution to the Theory of Economic Growth", Quarterly Journal of Economics 70, pp. 65-94.

17. Solow, R. (1974) "Intergenerational Equity and Exhaustible Resources", Review of Economic Studies 41 (Symposium on the Economics of Exhaustible Resources), pp. 29-45.

18. Stiglitz, J. (1974) "Growth with Exhaustible Resources: Efficient and Optimal Growth Paths", Review of Economic Studies 41 (Symposium on the Economics of Exhaustible Resources), pp.123138.

19. Tahvonen, O. \& Salo, S. 2001. "Economic Growth and Transitions between renewable and nonrenewable energy resources", European Economic Review 45, pp.13791398. 
Département des Sciences Économiques de l'Université catholique de Louvain

Institut de Recherches Économiques et Sociales

Place Montesquieu, 3

1348 Louvain-la-Neuve, Belgique 